\title{
FRACTIONAL PARTIAL DIFFERENTIAL EQUATIONS - A STUDY BY NUMERICAL METHODS
}

\section{BABU}

Assistant Professor, Department of Mathematics, Vels University, Pallavaram, Chennai, Tamil Nadu, India

This paper is to prove that, the fractional partial differential equation forms a finite domain with the numerical solution by using different fractional derivatives. The two fractions are used to prove the fractional diffusion equation and the fractional dispersion equation. The Fractional Differential Equation is formed from the standard diffusion equation $\beta \in\langle 0,1\rangle \in \in(0,1)$ replacing the order, as a second space derivative, with the fractional derivative $\alpha \in\langle 1,2\rangle$ $\alpha \in(1,2)$. The analytical solutions of, both the fractional diffusion equation and dispersion equation were derived. From these three numerical methods, the L1/L2-approximation method, the standard method, and the matrix transform method; only the third method is used to deal with the fractional derivative. The two fractional methods transmute into a system of ordinary differential equations that solves by the graphical method. It concludes by the numerical results, that have demonstrated the three numerical method's effectiveness and convergence.

KEYWORDS: Fractional Dispersion Equation, Fractional Derivative, L1/L2- Approximation Method, Standard Method, Matrix Transform Method \& Graphical Method.
\end{abstract}

Received: Jun 18, 2017; Accepted: Jun 28, 2017; Published: Jul 07, 2017; Paper Id.: IJMCARAUG20172

\section{INTRODUCTION}

Fractional derivative is not a new concept, but it is an old and familiar integer-order. The fractional derivatives were applied in many fields, like hydrology physics, biology, chemistry, finance, biochemistry, etc. This new fractional-order model is, more adequate than the initially used integer-order models, the reason is that, the fractional-order derivatives and integrals need the description of the memory and hereditary properties of different substances. This is the important advantage of the fractional-order models, when we compared with the neglected integer-order models.

In Physics, the classical Brownian motion model, spreads with a particle in an inconsistent way, when the fractional space derivatives are merged to, model anomalous diffusion or dispersion. Similarly, a left RiemannLiouville derivative and right Riemann-Liouville derivative, were used to allow the modeling of flow regime impacts, from either side of the domain. To model the transport of passive tracers, carried by fluid flow in a porous medium, for solute transport in a subsurface material dispersion equation, were used in groundwater hydrology. Green functions are difficult to evaluate but, the fractional-order partial differential equations, were analytically derived by it. It proves that, its great potential is helped to derive, the numerical treatment and it supports to analyze the fractional-order differential equations.

Probably, we cannot use the numerical method, to solve the fractional partial differential equation, with nonsymmetric fractional derivatives. The practical numerical method was used, to solve the one-dimensional space, with 
variable coefficients, on a finite domain. The backward differentiation formula was also used, to solve the space fractional equation, into a system of ordinary differential equations. The domain decomposition method and variation iteration method, are the two reliable developed algorithms, which are used to construct numerical solutions, that will be in the form of a rabidly convergent series, with computable components, so that, the theoretical analysis cannot be proved by that.

\section{THE FRACTIONAL DIFFUSION AND DISPERSIOIN EQUATION}

In this paper, the functionall partial differential equation forms a finite domain, with the numerical solution, by using different fractional derivatives.

$$
\begin{aligned}
& \frac{\partial u(x, t)}{\partial t}=K_{\alpha} \frac{\partial^{\alpha}}{\left.\left.\partial\right|_{x}\right|^{\alpha}} u(x, t)+K_{\rho} \frac{\partial^{\mu}}{\left.\left.\partial\right|_{x}\right|^{\beta}} u(x, t), \\
& 0<t \leq T, 0<x<L
\end{aligned}
$$

It is given by the initial boundary as

$$
u(0, t)=u(L, t)=0,
$$

$u(x, 0)=g(x)$

The solute concentration is ' $u$ '

$K_{\alpha} K_{\alpha}$ Is the dispersion coefficient

$K_{\beta} K_{\beta}$ Is the average fluid velocity

The Fractional derivatives of order $\alpha(1<\alpha \leq 2) \alpha(1<\alpha \leq 2)$ and $\beta(0<\beta \leq 2) \beta(0<\beta \leq 2)$ are defined as

$$
\begin{aligned}
& \frac{\partial^{\alpha}}{\partial|x|^{\alpha}} u(x, t)=-c_{\alpha}\left({ }_{0} D_{x}^{\alpha}+{ }_{x} D_{L}^{\alpha}\right) u(x, t) \\
& \frac{\partial^{\beta}}{\partial|x|^{\beta}} u(x, t)==-c_{\beta}\left({ }_{0} D_{x}^{\beta}+{ }_{x} D_{L}^{\beta}\right) u(x, t)
\end{aligned}
$$

Different techniques were used for different fractional derivatives, without confusing the remark that is essential to adopt the accuracy, for the corresponding definition of the fractional operator, for the standard accuracy and discreteness, of the fractional diffusion equation. The unstable finite difference scheme, results in finite difference method, as an explicit or an implicit one.

$$
\frac{\partial u(x, t)}{\partial t}=-K_{x}(-\Delta)^{\frac{\pi}{2}} u(x, t)-K_{\rho}(-\Delta)^{\frac{g}{2}} u(x, t)
$$

Fractional Laplacian operator and derivation are equivalent; this was widely assumed by the fractional derivative method. Firstly, the Laplacian was used by the Fourier transform, on an infinite domain with a natural extension, to include finite domains. The second is the eigenfunction expansion on a finite domain. The first operators are, indeed equivalent and the second is not mathematically equivalent because, it cannot derive the numerical approximations. The Fractional Diffusion equations, for numerical methods are: 


$$
\begin{aligned}
& \frac{\partial u(x, t)}{\partial t}=K_{\alpha} \frac{\partial^{\alpha}}{\partial|x|^{\alpha}} u(x, t), \\
& 0<t \leq T, 0<x<L, 1<\alpha \leq 2 \\
& u(0, t)=u(L, t)=0, \\
& u(x, 0)=g(x),
\end{aligned}
$$

\section{THE MATRIX TRANSFORM METHOD}

This method is used for the fractional diffusion equation, to propose with homogeneous boundary conditions. It shows that, the Matrix transform method gives the correct accurate analytic solution. This is formed by orthonormal eigenfunctions, from which the Matrix transform method and analytic solution are based on. In this subsection, the new technique can apply to the fractional diffusion equation, based on orthonormal eigenfunctions. The matrix is also a symmetric positive definite, which exist here as a nonsingular matrix.

Even the matrix is an eigenvector, symmetric tridiagonal matrix and eigenvalues which can be formed in an easiest method. Numerical examples with a finite domain are:

$$
\begin{aligned}
& \frac{\partial u(x, t)}{\partial t}=-K_{\alpha} \frac{\partial^{\alpha}}{\partial|x|^{\alpha}} u(x, t), \\
& 0<t \leq T, 0<x<\pi, \quad 1<\alpha \leq 2 \\
& u(x, 0)=x^{2}(\pi-x), \\
& u(0, t)=u(\pi, t)=0 . \\
& u(x, t)=\sum_{n=1}^{\infty}\left[\frac{8}{n^{3}}(-1)^{n+1}-\frac{4}{n^{3}}\right] \sin (n x) \\
& \exp \left(-\left[K_{\alpha}\left(n^{2}\right)^{\frac{\alpha}{2}}+K_{\beta}\left(n^{2}\right)^{\frac{\beta}{2}}\right] t\right) .
\end{aligned}
$$

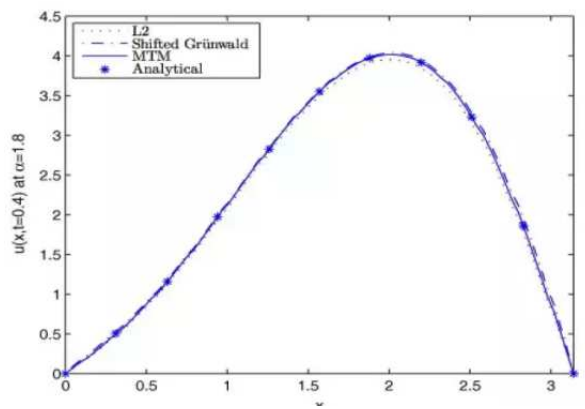

Figure 1

In this figure 1, the analytic solution and the solutions obtained by the third numerical method is given. From this it can be seen that, the matrix numerical solution is in good agreement with the analytic solution. 


\section{ANALYTIC SOLUTION FOR MATRIX TRANSFORM METHOD}

The Matrix Transform Method, henceforth, as MTM and analytic solution are based on orthonormal eigenfunctions, but only the maximum errors of the MTM is present here. The backward differentiation formulas are used to solve the ordinary differential equations, by using transformed fractional equation.

From the Table 1 and Table 2, it shows the maximum errors of the MTM, for solving the fractional partial differential equation. The MTM is stable and convergent for solving the fractional diffusion equation, based on orthonormal eigenfunctions. The analytical solutions of the fractional partial differential equations are derived by L1 and L2 Matrix Transform Method, which is difficult to evaluate.

Table 1: Errors of L1 MTM

\begin{tabular}{|c|c|}
\hline $\mathbf{h}=\pi / \mathbf{n}$ & MTM L1 \\
\hline$\pi / 10$ & $2.217 \mathrm{E}-2$ \\
\hline$\pi / 20$ & $5.759 \mathrm{E}-3$ \\
\hline$\pi / 40$ & $1.481 \mathrm{E}-3$ \\
\hline$\pi / 80$ & $3.727 \mathrm{E}-4$ \\
\hline
\end{tabular}

Table 2: Errors of L2 MTM

\begin{tabular}{|c|c|}
\hline $\mathbf{h}=\pi / \mathbf{n}$ & MTM L2 \\
\hline$\pi / 10$ & $1.9963 \mathrm{E}-2$ \\
\hline$\pi / 20$ & $5.1683 \mathrm{E}-3$ \\
\hline$\pi / 40$ & $1.3386 \mathrm{E}-3$ \\
\hline$\pi / 80$ & $3.3519 \mathrm{E}-4$ \\
\hline
\end{tabular}

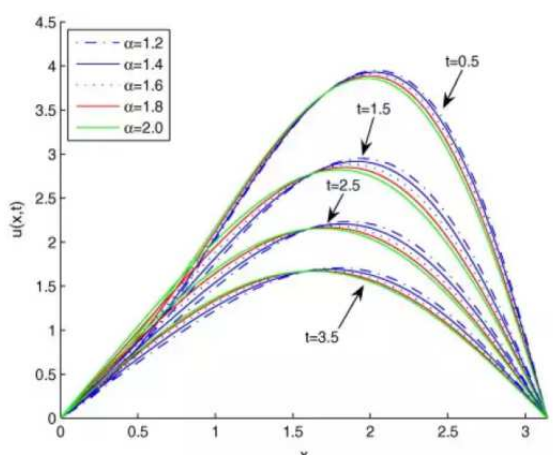

Figure 2

The numerical examples of fractional partial differential equation on a finite domain are:

$$
\begin{aligned}
& \frac{\partial u(x, t)}{\partial t}=K_{\alpha} \frac{\partial^{\alpha}}{\partial|x|^{\alpha}} u(x, t), \\
& 0<t \leq T, 0<x<\pi, \\
& 1<\alpha \leq 2 \\
& u(0, t)=\sin 4 x, \\
& u(0, t)=u(\pi, t)=0 .
\end{aligned}
$$


By demonstrating the calculation of these two methods, we can consider the following Table 3 , for the maximum errors of the standard method, to solve the fractional diffusion equation and the fractional dispersion equation.

Table 3: Errors of Standard Method

\begin{tabular}{|c|c|c|}
\hline $\mathbf{H}=\mathbf{1} / \mathbf{N}$ & $\mathbf{A 1} / \mathrm{A} 2$ & $\begin{array}{c}\text { Standard } \\
\text { Method }\end{array}$ \\
\hline $1 / 50$ & $\begin{array}{c}1.8144 \mathrm{E}- \\
2\end{array}$ & $2.8191 \mathrm{E}-3$ \\
\hline $1 / 100$ & $\begin{array}{c}9.4917 \mathrm{E}- \\
3\end{array}$ & $1.5093 \mathrm{E}-3$ \\
\hline $1 / 200$ & $\begin{array}{c}4.8584 \mathrm{E}- \\
3\end{array}$ & $7.7821 \mathrm{E}-4$ \\
\hline $1 / 400$ & $\begin{array}{c}2.4586 \mathrm{E}- \\
3\end{array}$ & $3.9459 \mathrm{E}-4$ \\
\hline
\end{tabular}

The impact of fractional order is again demonstrated, which is the example of the RFADE, with a different condition.

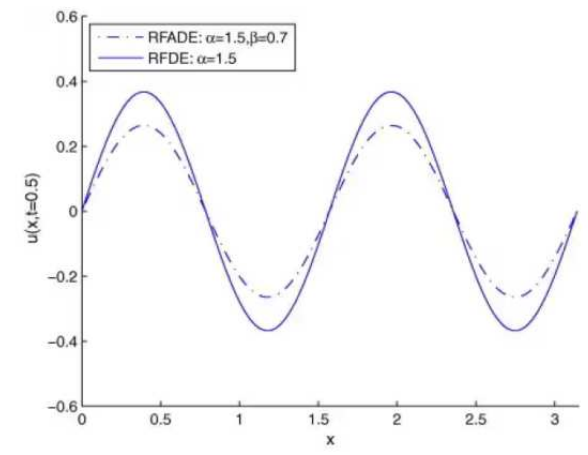

Figure 3

Figure 3, is a comparison of the fractional diffusion equation and the fractional dispersion equation, with a finite domain.

\section{CONCLUSIONS}

This paper concludes that, the third effective numerical method to solve the fractional diffusion equation and the fractional dispersion equation, on a finite domain, with orthonormal Eigen functions, has been described. Two methods, which were defined by the fractions, were discussed. The Numerical method's results have demonstrated the effectiveness and convergence of the Matrix Transform methods. The methods and techniques, which were discussed in this paper, can be easily applied to solve the fractional partial differential equations, even in other kinds.

\section{REFERENCES}

1. Changpin, Li, Fanhai Zeng. Numerical Methods for Fractional Calculus. Taylor and Francis Publication, 2015.

2. Gear, C, W. The automatic integration of ordinary differential equations. Volume 4, Issue 3, 1971, pp. 176-179

3. Heinbockel, J, H. "Orthogonal Functions". Numerical Methods for Scientific Computing. London: Trafford Publication, 2004.

4. Heinbockel, J, H. "Partial Differential Equations". Numerical Methods for Scientific Computing. London: Trafford Publication, 2004. 
5. Miller K, S. An Introduction to the Fractional Differential Equation. New York: Marcel Dekker, 1997.

6. Metzler, R, J, Klafter. “Boundary value problems for fractional diffusion equations”. Physica A: Staistics. Appl., Volume 278, Issue 1-2, 2000, pp. 107-125.

7. Momani, S, Z, Odibat. "Numerical solutions of the fractional advection-dispersion equation". Numerical Methods a Complete study. Volume 24, Issue 6, 2008, pp. 1416-1429.

8. Meerschaert, M, M. "Finite difference methods for two-dimensional equation". J. Comput. Phys., Volume 211, Issue 1, 2006, pp. 249-261

9. Pettofrezzo, J, Anthony. Matrices and Transformations. New York: Dover Publications, 1978. 